\title{
Dosage compensation and the global re-balancing of aneuploid genomes
}

\author{
Matthias Prestel, Christian Feller and Peter B Becker*
}

\begin{abstract}
Diploid genomes are exquisitely balanced systems of gene expression. The dosage-compensation systems that evolved along with monosomic sex chromosomes exemplify the intricacies of compensating for differences in gene copy number by transcriptional regulation.
\end{abstract}

Complex genomes are more than just the sum of their genes, but are rather complex regulatory systems in which the expression of each individual gene is a function of the activity of many other genes, so that the levels of their protein products are maintained within a narrow range. Such homeostasis favors the maintenance of the appropriate stoichiometry of subunits in multiprotein complexes or of components in signal transduction pathways, and defines the 'ground state' of a cell [1]. In diploid genomes, both alleles of a gene are usually active and this 'double dose' of each gene is figured into the equation. Thus, deviations from diploidy, such as the deletion or duplication of genes or of larger chromosomal fragments (aneuploidy), unbalance the finely tuned expression of the genome. Segmental aneuploidies of this kind can arise from failed or faulty repair of chromosomal damage due to irradiation, chemical insult or perturbation of replication, or from illegitimate recombination during meiosis. Loss or duplication of entire chromosomes (monosomy or trisomy, respectively) can arise from nondisjunction during cell division. Depending on the extent of the aneuploidy and on the genes affected, the fine balance of trans-acting factors and their chromosomal binding sites that define the gene-expression system is disturbed, and the fitness of the cell or organism challenged.

Often, aneuploidies have been associated with a variety of developmental defects and malignant aberrations,

*Correspondence: pbecker@med.uni-muenchen.de

Adolf-Butenandt-Institute and Centre for Integrated Protein Science (CiPSM), Ludwig-Maximilians-University, Schillerstrasse 44, 80336 Munich, Germany such as Down syndrome or certain breast cancers (reviewed in $[2,3]$ ). The phenotypes associated with changes in gene copy number can not only be the result of the deregulation of the affected gene(s), but may also reflect trans-acting effects on other chromosomal loci or even more global alterations of the entire regulatory system. This is particularly true if genes coding for regulatory factors, such as transcription factors, are affected (reviewed in $[4,5]$ ).

\section{Strategies for re-balancing aneuploid genomes}

Genome-wide studies in different organisms reveal that the expression of a substantial number of genes directly correlates with gene dose (the primary dosage effect) [6]. In other cases, the measured expression levels do not reflect the actual copy number, as compensatory mechanisms aimed at re-establishing homeostasis take effect $[4,5]$. Imbalances due to aneuploidy may be compensated for at any step of gene expression from transcription to protein stability. Excess subunits of multiprotein complexes that are not stabilized by appropriate interactions are susceptible to degradation (see [1] for a discussion of compensation at the protein level). Dosagecompensation mechanisms at the level of transcription are versatile, intricate, and in no instance are they fully understood.

In principle, three types of compensatory responses to aneuploidies are recognized: buffering, feedback, and feed-forward, which may act individually or, more likely, in combination [7]. Oliver and colleagues [7] define buffering as 'the passive absorption of gene dose perturbations by inherent system properties'. Currently, the nature of this general or 'autosomal' buffering is unknown, but its existence can be deduced from comparing gene expression to DNA copy number in healthy and aneuploid genomes [8-11]. The system properties referred to by Oliver and colleagues can be considered as the sum of the biochemical equilibria of the system living cell', which are predicted to moderate the effect of the reduction of one component. Apparently, the deletion of one gene copy (that is, a twofold reduction in gene expression) can be partially compensated for by increasing the steady-state mRNA levels originating from 
the remaining allele by, on average, 1.5-fold [7,11]. Interestingly, Stenberg and colleagues [11] observed that buffering appears to compensate for deficiencies better than for gene duplications, which leaves open the existence of a general sensor of monosomy that mediates the effect. A general buffering will also ameliorate the consequences of widespread mono-allelic gene expression due to parental imprinting (cases where a single allele is expressed, depending on whether it is inherited from the father or mother) [12].

In contrast to the general and nonspecific buffering just described, a 'feedback' mechanism would be defined as gene-specific - sensing and readjusting the levels of specific molecules by appropriate, specific mechanisms. Finally, 'feed-forward' anticipates the deviation from the norm and hence can only be at work in very special circumstances. Prominent examples where feed-forward scenarios are applicable are the widely occurring monosomies in the sex chromosomes of heterogametic organisms (for example, the XX/XY sex-chromosome system), which are present in each and every cell of the species.

In contrast to aneuploidies that arise spontaneously, these 'natural' monosomies and their associated dosagecompensation mechanisms are the products of evolution. Research on dosage-compensation mechanisms associated with sex chromosomes continues to uncover unexpected complexities and intricacies. The somatic cells of the two sexes of the main model organisms of current research - mammals, nematode worms (Caenorhabditis elegans) and fruit flies (Drosophila melanogaster) - differ in that those of females are characterized by two $\mathrm{X}$ chromosomes, while those of males have one $\mathrm{X}$ and one Y chromosome (mammals and Drosophila); or one sex $(\mathrm{XX})$ is a hermaphrodite and the males have just a single $\mathrm{X}$ and no $\mathrm{Y}$ chromosome (X0) (C. elegans) [13]. Remarkably, different dosage-compensation strategies for balancing gene expression from the $\mathrm{X}$ chromosome between the sexes have evolved independently in these three cases (Figure 1), as we shall discuss in this article. There is increasing evidence that in all three cases, the transcription of most genes on the single male $\mathrm{X}$ chromosome is increased roughly twofold [14-16]. In fruit flies, this upregulation of the $\mathrm{X}$ chromosome is limited to males. In mammals and worms, however, the $\mathrm{X}$ chromosomes appear to be also upregulated in the XX sex, which necessitates additional compensatory measures. In female mammals, one of the $\mathrm{X}$ chromosomes is globally silenced, whereas in hermaphrodite worms, gene expression on both $\mathrm{X}$ chromosomes is downregulated by about 50\% (Figure 1). An emerging principle is that the net fold-changes of dosage compensation are not achieved by a single mechanism (that is, there is no simple switch for 'twofold up'), but by integration of activating and repressive cues, as discussed later.

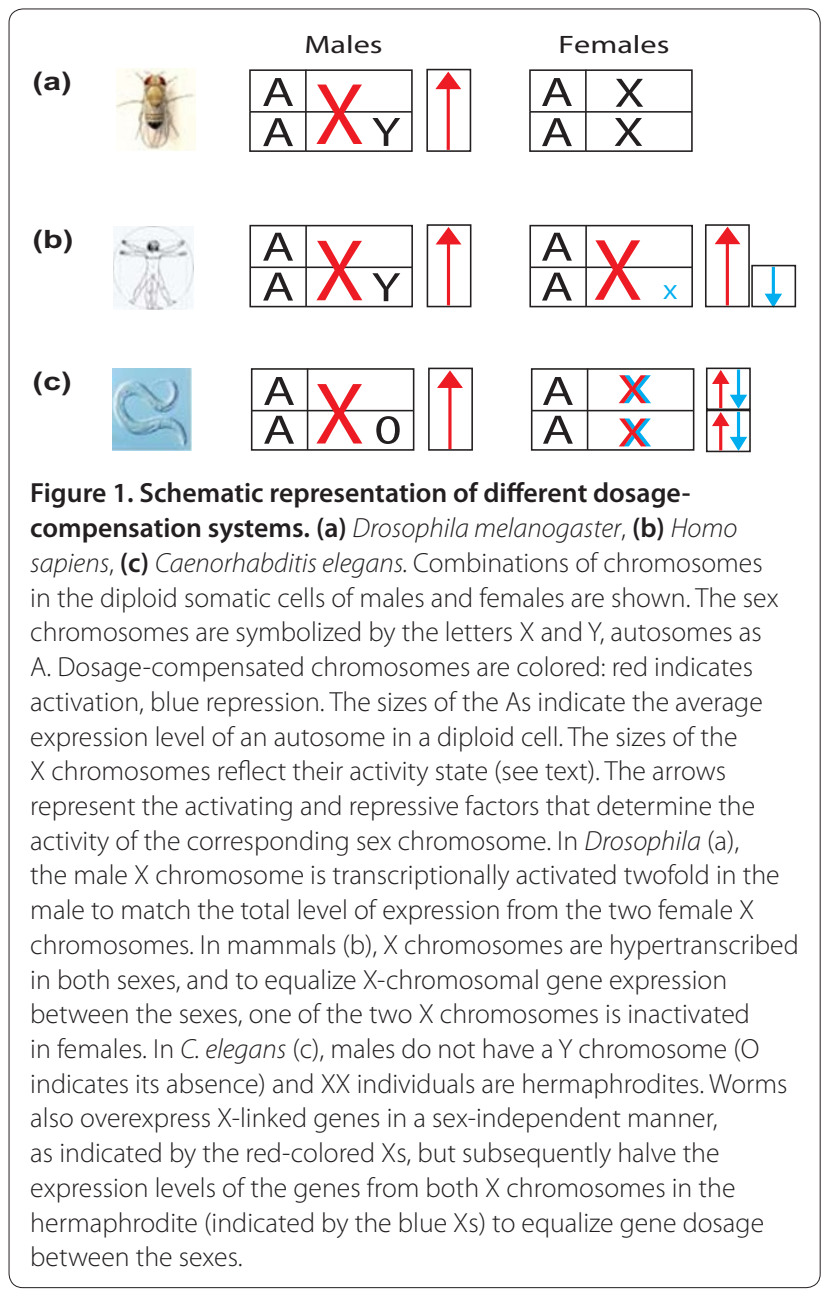

In what follows we summarize recent insight into the dosage-compensation mechanisms of the XX/XY sex chromosome systems, which nicely illustrate the evolution of global, genome-wide regulatory strategies. However, compensation systems of this type are not absolutely required for the evolution of heterogametic sex. Birds, some reptiles, and some other species use the ZW/ZZ sex-chromosome system, which does not use the mechanism of chromosome-wide transcriptional regulation to compensate for monosomy [17-19].

\section{Dosage compensation of sex chromosomes reveals the balancing capacity of chromatin}

The sex chromosomes of the XX/XY system are thought to have originated from two identical chromosomes in a slow process that was initiated by the appearance of a male-determining gene. In order to be effective, this gene should be propagated only in males, which was achieved by evolving a Y chromosome that was specifically propagated through the male germline. The necessary suppression of recombination between this 'neo-Y' chromosome 
and the corresponding sister chromosome (which would become the future 'neo-X') favored the accumulation of mutations, deletions and transposon insertions, an erosive process that led to loss or severe degeneration of $Y$ chromosomes [20-24]. The progressive erosion of the evolving Y left many X-chromosomal genes without a corresponding copy on the $\mathrm{Y}$ chromosome (the hemizygous state). The initial consequences of gene loss on the $\mathrm{Y}$ chromosome may have been absorbed by the intrinsic biochemical buffering properties of the cell noted above [11]. However, when the majority of genes on the $\mathrm{X}$ chromosome lost their homologs on the $\mathrm{Y}$ chromosome the co-evolution of regulatory processes to overcome the reduced gene dose - that is, dosagecompensation systems - increased the fitness of the organisms. These dosage-compensation systems are likely to originate in the male sex (XY or X0 in the examples discussed here), as it is in males that factors acting in a dose-dependent manner (such as transcription factors, chromatin constituents and components of signaltransduction cascades) would become limiting $[25,26]$.

A logical adaptation to ensure the survival of males would be the increased expression of X-chromosomal genes [6]. This intuitively obvious mechanism has long been known in Drosophila. Observing the specialized polytene chromosomes in larvae (which are composed of thousands of synapsed chromatids arising from repeated DNA replication without chromosome segregation), Mukherjee and Beermann [27] were able to directly visualize nascent RNA and found that the single X chromosome in males gave rise to almost as much RNA as two autosomes. Recent genome-wide expression analyses confirmed these early observations $[28,29]$ and further genome-wide studies suggest that this mechanism may also operate in C. elegans and mammals [14-16]. For these species neither the mechanism of this chromosomewide regulation nor the factors involved are known.

For Drosophila, however, thanks to decades of outstanding genetics exploring male-specific lethality, we know at least a few of the prominent players. Here, the twofold stimulation of transcription on the $\mathrm{X}$ chromosome is mediated by the male-specific assembly of a dosage-compensation complex (the Male-Specific-Lethal (MSL) complex), a ribonucleoprotein complex that associates almost exclusively with the $\mathrm{X}$ chromosome (reviewed in [30]; Figure 2). Most subunits of the MSL complex are found in both sexes of Drosophila, except for the key protein MSL2 and the noncoding roX (RNA-onthe-X) RNAs, which are only expressed in males (Figure 2), thus leading to the assembly of the MSL complex exclusively in male cells. The MSL complex associates with the transcribed regions of target genes in a multistep process that has been reviewed elsewhere [31-33]. Key to the stimulation of transcription is the
MSL-complex subunit MOF (Males-absent-on-the-first; also known as KAT8, lysine acetyltransferase 8), a histone acetyltransferase with specificity for lysine 16 in the amino-terminal tail of histone 4 (H4K16ac). Acetylation of this residue is known to reduce interactions between nearby nucleosomes and leads to unfolding of nucleosomal fibers in vitro [34,35].

Whereas the action of the dosage-compensation complex in Drosophila is limited to males, in C. elegans and mammals the unknown factors that stimulate Xchromosomal transcription appear to be active in the hermaphrodite and the female, as well as in males. If, however, $\mathrm{X}$ activation re-balances the male genome in these species, it follows that in the XX sex, having two hyperactive $\mathrm{X}$ chromosomes relative to the autosomes must be suboptimal [36]. Consequently, further compensation is needed. Mammals have evolved a strategy of inactivating one of the female $\mathrm{X}$ chromosomes to achieve a level of X-chromosome gene expression closely resembling that from the single $\mathrm{X}$ in males (reviewed in [37]; Figure $1 \mathrm{~b}$ ). Which $\mathrm{X}$ is inactivated is random, and inactivation starts with the stable transcription of the long, non-coding Xist (Xi-specific transcript) and RepA (repeat A) RNAs from a complex genetic region on the future inactive $\mathrm{X}(\mathrm{Xi})$ called the $\mathrm{X}$-inactivation center. Subsequently, Xist RNA - possibly in complex with undefined protein components - spreads to coat the entire Xi. Silencing involves the recruitment and action of the Polycomb silencing machinery via the Xist and RepA RNAs [38,39], followed by reinforcement through the incorporation of histone variants, removal of activating histone modifications and DNA methylation [37]. Remarkably, the independent evolution of nematode worms arrived at a very different solution to the problem. $C$. elegans equalizes the gene dose by halving the expression levels of genes on both $\mathrm{X}$ chromosomes in the hermaphrodite, using a large dosage-compensation complex containing components of the meiotic/mitotic condensin. The involvement of condensins may point to regulation at the level of chromatin fiber compaction ([40] and references therein). The scenario shown schematically in Figure 1c for C. elegans suggests that dosage compensation in this species involves a twofold increase in X-linked transcription in both sexes, which is opposed by a twofold repression in hermaphrodites. The underlying mechanisms are still mysterious.

This short summary of the three very different dosagecompensation systems reveals two common denominators. First, they all adapt factors and mechanisms, which are already involved in other regulatory processes, for the compensation task by harnessing them in a new molecular context. Furthermore, these factors are all known for their roles in modulating chromatin structure. It seems that chromatin can adopt a variety of structures 


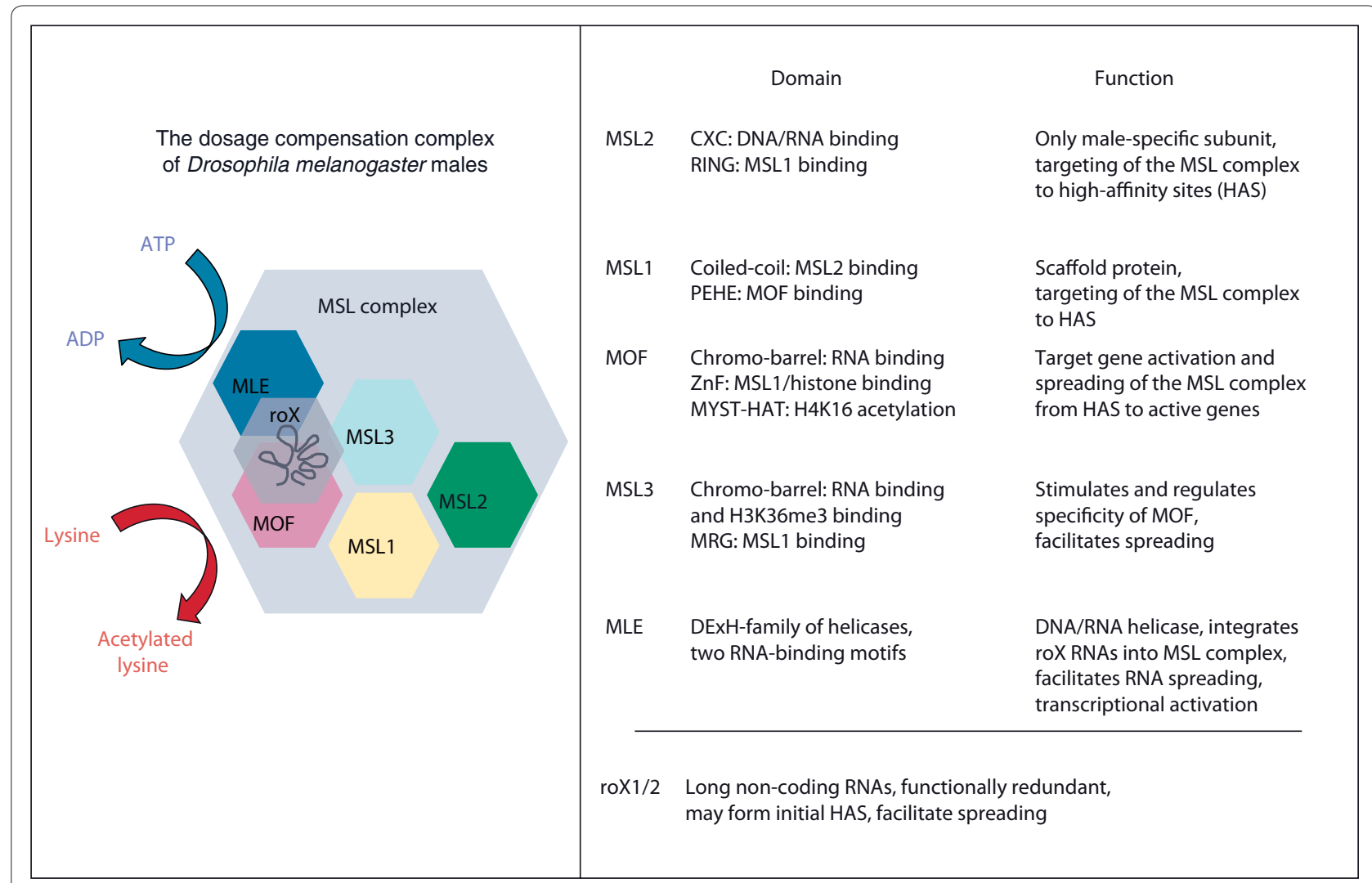

Figure 2. The Drosophila melanogaster male dosage-compensation complex. The complex, called the MSL complex in Drosophila, consists of five proteins (MSL1, MSL2, MSL3, MOF, MLE) and two non-coding roX RNAs. The proteins, but not the roX RNAs, are evolutionarily conserved, as related proteins can be found in yeast and humans (for details see $[30,68,69])$. The box lists the conserved protein domains of the individual members of the Drosophila MSL complex and their identified functions for dosage compensation. MSL2 is the only male-specific protein subunit; all other subunits are present in both sexes. The two roX RNAs (see bottom of table) are also only expressed in males. The curved arrows symbolize the known enzymatic activities in the dosage-compensation complex. MLE is an RNA helicase that hydrolyzes ATP to effect conformational changes in DNA and RNA [70]. MOF is a lysine acetyltransferase with specificity for lysine 16 of histone H4. Abbreviations of the protein domains are: CXC, cysteine-rich domain; ZnF, zinc finger; PEHE, proline-glutamic acid-histidine-glutamic acid; HAT, histone acetyltransferase; MYST, MOZ (monocytic leukemia zinc finger protein), YBF2/SAS3 (something about silencing 3), SAS2 and TIP60 (60 kDa Tat-interactive protein); MRG, mortality factor on chromosome 4 related gene and DExH, aspartic acid-glutamic acid-x-histidine.

with graded activity states, which can be used either to completely switch off large chromosomal domains or to fine-tune transcription (either up or down) in the twofold range. Dosage compensation therefore integrates with other aspects of chromatin organization. In Drosophila, the male X chromosome that accumulates the H4K16 acetylation mark is particularly sensitive to mutations in general chromatin organizers. Prominent among these is the zinc finger protein $\mathrm{Su}(\mathrm{var}) 3-7$ (suppressor of variegation 3-7), a heterochromatin constituent known to bind HP1 (heterochromatin protein 1). Normal levels of $\mathrm{Su}$ (var)3-7 are required for proper dosage compensation and to ensure the selective binding of the dosagecompensation complex to the $\mathrm{X}$ chromosome [41-43]. The male $\mathrm{X}$ polytene chromosome bloats when $\mathrm{Su}(\mathrm{var}) 3-7$ levels are reduced and condenses when the protein is in excess. These changes in chromatin condensation depend on a functional dosage compensation complex, suggesting that the MOF-catalyzed acetylation of histone 4, and subsequent unfolding effect of H4K16ac, is constrained by as yet unknown counteracting factors (Figure 3a), conceivably by ones that promote chromatin compaction.

Selective, massive unfolding of the dosage-compensated male X chromosome in Drosophila is also observed when the nucleosome remodeling factor (NURF) is inactivated $[44,45]$. Nucleosome remodeling by NURF may thus also serve to counteract excessive unfolding due to H4K16 acetylation. Tamkun and colleagues [46] suggested that NURF might achieve this task by maintaining sufficiently high histone $\mathrm{H} 1$ levels on the $\mathrm{X}$ chromosome. Clearly, the degree of chromatin compaction can be adjusted by integration of unfolding and compacting factors. 


\section{Harnessing MOF for dosage compensation}

Further analysis of the role of Drosophila MOF in dosage compensation suggests that it may affect gene expression by modulating the productivity of the transcription machinery in the chromatin context. Although MOF is able to acetylate non-histone substrates $[47,48]$, its main substrate in the context of dosage compensation is the strategic H4K16. Biochemical studies showed that this modification interferes directly with the folding of the nucleosomal chain into 30-nm fibers in vitro [35,49]. Accordingly, H4K16 acetylation by MOF has the potential to counteract chromatin-mediated transcriptional repression [50,51] (Figure 3a). In the simplest scenario, the only task of the MSL complex in Drosophila would be to enrich MOF on the X chromosome relative to the autosomes. However, studies of the effect of MOF in yeast or in a cell-free chromatin transcription system showed that H4K16 acetylation does not automatically increase transcription by twofold, but by many-fold [50]. This strong activation potential of MOF can also be visualized in Drosophila. We recently established Drosophila lines in which MOF is tethered to a $\beta$-galactosidase reporter gene engineered to reside on an autosome [51]. Sorting adult flies according to sex allowed comparison of MOF-dependent reporter gene stimulation in male flies, where MOF is part of the dosage-compensation complex, and in females, where its molecular context was initially unknown. In females, MOF recruitment stimulated transcription from a proximal promoter by an order of magnitude. The effect faded with increasing distance between recruitment site and transcription start site and therefore appears to be related to local chromatin opening by promoter-bound co-activators.

By contrast, the molecular context of the MSL complex in males restricted the activation effect of MOF to the twofold range reminiscent of dosage compensation, and this effect was observable over a distance of $5 \mathrm{~kb}$ [51]. Notably, similar H4K16 acetylation levels accompanied the very different activation modes in the two sexes. So it seems that the activation potential of H4K16 acetylation revealed in females is constrained in males. Ectopic assembly of the MSL complex in females by expression of MSL2 constrained the strong activation to a twofold range [51]. We concluded from these and further studies that the Drosophila dosage-compensation complex achieves a twofold activation of transcription by integrating activating and repressive principles [51].

MOF serves as an example of the principle that dosage compensation employs chromatin modifiers that are also functional in other contexts. MOF is expressed at only slightly lower levels in females than in males, and it also resides in at least one other complex in addition to the MSL complex. Mendjan et al. [52] first reported the existence of an alternative complex (the NSL complex,
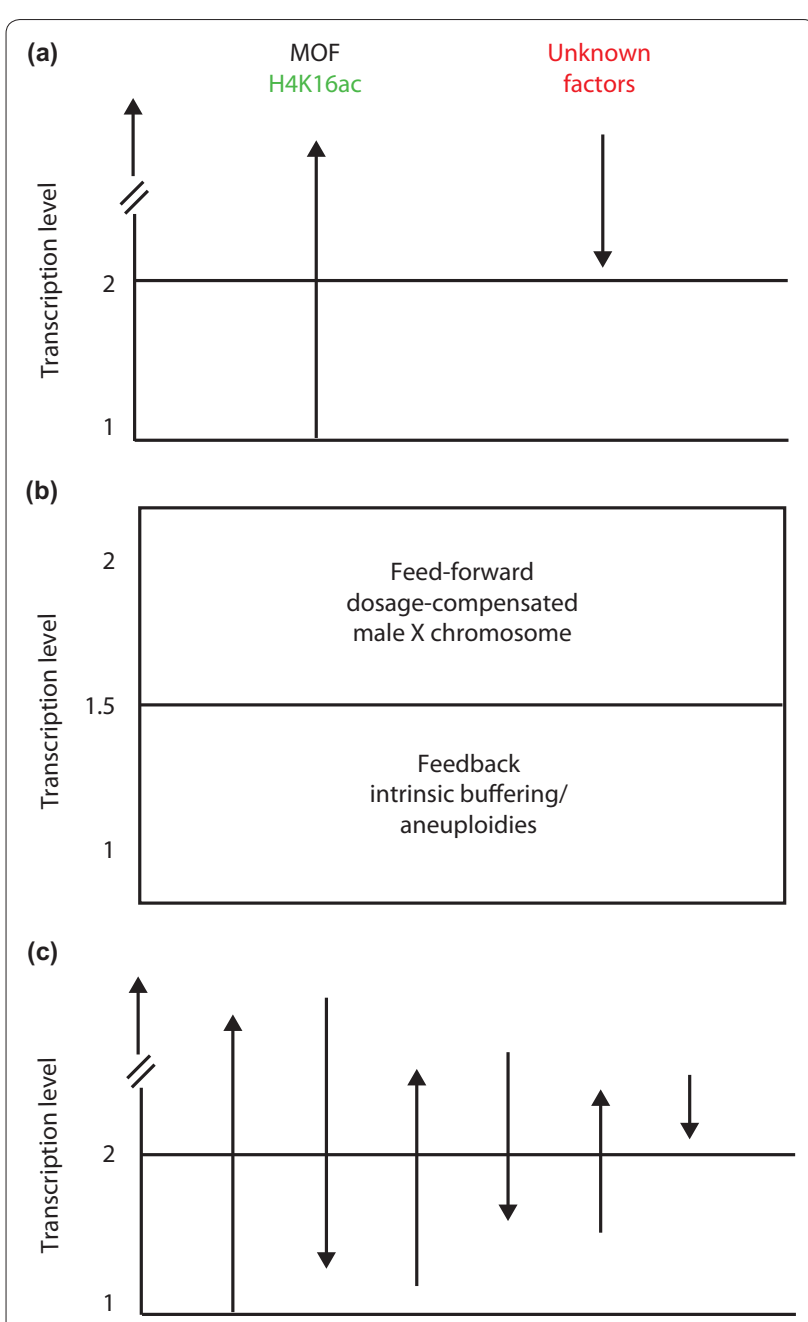

Figure 3. Possible mechanisms for dosage compensation. (a) The twofold activation of the single male $X$ chromosome in Drosophila could be achieved by a large, MOF-dependent activation of transcription through H4K16 acetylation and its counteraction by yet unknown factors, mediated by the dosage-compensation complex in males [51]. In (a,b), transcriptional level 1 refers to the normal regulated level of transcription from a single uncompensated $X$ chromosome in females. (b) Furthermore, the twofold activation of the male $X$ chromosome could be achieved by a combination of mechanisms: a general buffering/feedback component and a dedicated feed-forward mechanism (dosage compensation as suggested in (a)) [7]. The effects of these two processes could add up to the expected twofold compensation required to equalize the expression of $\mathrm{X}$-linked genes between the sexes. (c) Precise transcription levels could result from negotiation between a number of activating and repressive factors (up and down arrows). In this instance, transcriptional level 1 refers to a 'basal' transcription state. This hypothetical model assumes that additional factors beyond those mentioned in (a) and (b) contribute to final transcription levels, such as male-enriched protein kinases, heterochromatin components, chromatin remodelers, and others (for details, see text).

for 'Non-Specific-Lethal') in mixed-sex embryos and male cells of Drosophila, which contained a number of poorly characterized nuclear proteins and two 
components of nuclear pores [52]. The closely related MOF-MBD-R2 complex, purified by us from female Drosophila cells [51], shares several prominent components with the NSL complex, including WDS (Will Die Slowly, a homolog of mammalian WDR5 (WD repeatcontaining protein 5), dMCRS2 (microspherule protein 1), a forkhead-associated domain protein, and MBD-R2 (an uncharacterized protein harboring similarity to methylCpG-binding domains) [53]. In contrast to the NSL complex, the MOF-MBD-R2 complex does not contain nuclear pore components [51].

The evidence so far suggests that the MOF-MBD-R2 complex provides the molecular context for the strong activation elicited by MOF in females. Globally, MOF colocalizes with MBD-R2 to active genes with enrichment towards their 5' ends on all chromosomes in male and females, except for the male X chromosome (Figure 4). In male Drosophila cells, MOF is enriched on the $\mathrm{X}$ chromosome, where it co-localizes with MSL-complex components (such as MSL1) with a bias towards the 3' end (Figure 4). In male Drosophila cells, MOF apparently distributes dynamically between the two complexes. Ectopic expression of MSL2 in female cells, which leads to assembly of a dosage-compensation complex, relocalizes MOF from the autosomes to the $\mathrm{X}$ chromosome and from the $5^{\prime}$ end to the $3^{\prime}$ end of transcribed genes. The 3' enrichment suggests that dosage compensation in Drosophila may act at the level of transcription elongation $[54,55]$.

The earlier notion that MOF, a global activator of transcription, was harnessed to balance the X-chromosomal monosomy in male Drosophila is supported by the fact that the H4K16-specific acetyltransferase activity has been conserved during evolution, although its biological function has not $[56,57]$. MOF (KAT8) is the best-studied member of the evolutionarily conserved family of MYST acetyltransferases (MOZ (monocytic leukemia zinc finger protein), YBF2/SAS3 (something about silencing 3), SAS2 and TIP60 (60 kDa Tat-interactive protein)). To the best of our knowledge, mammalian MOF is not involved in dosage compensation, but in regulating gene expression in more specific ways and in maintaining genome stability. Knock-down of human MOF impairs the signaling of DNA damage via the ATM pathway in response to double-strand breaks, causing increased cell death and a loss of the cell-cycle checkpoint response [58]. Mouse MOF is essential for oogenesis and embryogenesis [59]. Loss of H4K16ac is a cancer hallmark [60] and MOF is deregulated in a number of diseases $[61,62]$.

As in Drosophila, mammalian MOF resides in several distinct complexes. These include the MOF-MLL1-NSL complex, which is required for the expression of the Hox 9a gene [63]; a complex containing the homologs of the Drosophila MSL3 and MSL1 that contributes to global

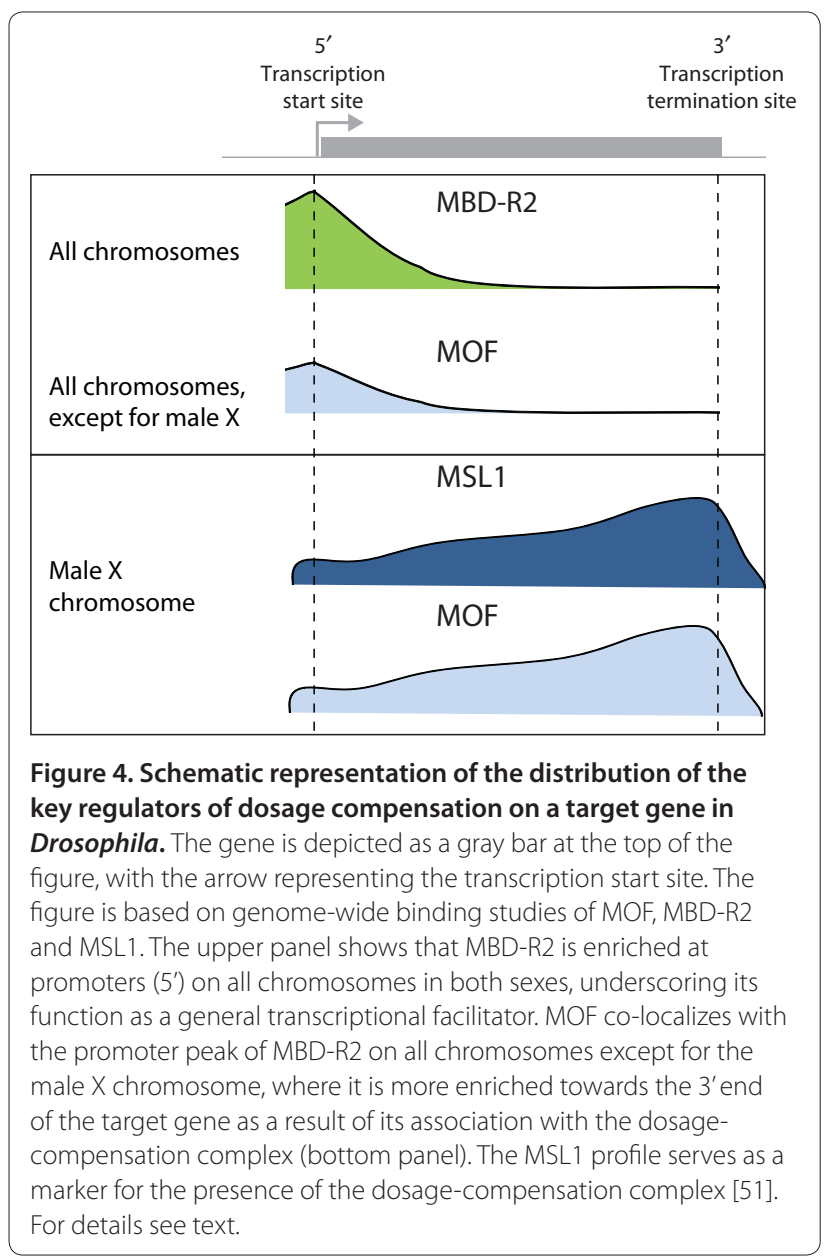

H4K16 acetylation [64,65]; and a complex most closely related to the Drosophila NSL complex [52], containing human NSL1 (MSL1v1) and PHF20 (PHD finger protein 20, the homolog of MBD-R2), in addition to other NSL protein homologs. This complex has attracted particular attention as it is not only responsible for the majority of H4K16ac in human cells [66], but also acetylates p53 at lysine 120 (K120) [66,67]. p53 in which K120 is mutated can no longer trigger the apoptotic pathway, yet its role in the cell-cycle checkpoint is not impaired. Evidently, the substrate specificity of human MOF and the physiological processes in which it is involved are largely determined by the molecular context of the acetyltransferase, defined by the composition of the different complexes. In Drosophila, however, one of the complexes has been adapted to serve the goal of balancing the genome for dosage compensation.

\section{Negotiation for small effects}

Although the mechanisms through which aneuploidies are compensated for are still mysterious, a number of overarching principles have emerged during recent years, 
mainly through studies of the X-chromosome monosomies. First, there is no simple switch for 'two-fold up' or 'two-fold down'. Optimal expression levels are negotiated by opposing principles. The X-chromosomal expression in hermaphrodite $C$. elegans results from integration of a global, twofold increase in expression in both sexes and a different counteracting hermaphroditespecific principle, which halves the expression again (Figure 1c).

The first genome-wide comparison of copy number and transcription in Drosophila revealed that a local or chromosomal hemizygosity is compensated for by the integration of at least two different mechanisms: an approximately 1.5 -fold compensation can be attributed to general buffering or feedback effects, whereas the remaining compensation is contributed by the evolution of a feed-forward mechanism involving a dedicated dosage-compensation complex [7] (Figure 3b). Furthermore, the twofold activation in male Drosophila is a composite of a much larger stimulation, which is opposed by a repressive principle (Figure $3 \mathrm{a}$ ). We therefore envisage that adjustment of the optimal gene expression levels may be a consequence of negotiation between a number of counteracting activating and repressing principles (Figure 3c). The complex and layered organization of chromatin appears to us as an advanced equalizer with many levers to allow optimal tuning of the transcription melody.

\section{Acknowledgements}

This work was supported by the Deutsche Forschungsgemeinschaft through SFB-TR5 and the Gottfried-Wilhelm-Leibniz Program. We thankT Straub, C Regnard and T Fauth for comments that improved the manuscript. CF is a fellow of the International Max-Planck Research School in Munich.

\section{Published: 26 August 2010}

\section{References}

1. Veitia RA, Bottani S, Birchler JA: Cellular reactions to gene dosage imbalance: genomic, transcriptomic and proteomic effects. Trends Genet 2008, 24:390-397.

2. Schinzel A: Catalogue of Unbalanced Chromosome Aberrations in Man. Berlin: Walter de Gruyter; 2001.

3. Bannon JH, McGee MM: Understanding the role of aneuploidy in tumorigenesis. Biochem Soc Trans 2009, 37:910-913.

4. Birchler JA: Reflections on studies of gene expression in aneuploids. Biochem J 2010, 426:119-123.

5. Deng X, Disteche CM: Genomic responses to abnormal gene dosage: the $X$ chromosome improved on a common strategy. PLOS Biol 2010, 8:e1000318.

6. Zhang Y, Oliver B: Dosage compensation goes global. Curr Opin Genet Dev 2007, 17:113-120.

7. Zhang Y, Malone JH, Powell SK, Periwal V, Spana E, Macalpine DM, Oliver B: Expression in aneuploid Drosophila s2 cells. PLOS Bio/ 2010, 8:e1000320.

8. FitzPatrick DR: Transcriptional consequences of autosomal trisomy: primary gene dosage with complex downstream effects. Trends Genet 2005, 21:249-253.

9. Aï Yahya-Graison E, Aubert J, Dauphinot L, Rivals I, Prieur M, Golfier G, Rossier J, Personnaz L, Creau N, Bléhaut H, Robin S, Delabar JM, Potier MC: Classification of human chromosome 21 gene-expression variations in Down syndrome: impact on disease phenotypes. Am J Hum Genet 2007 81:475-491.

10. Makarevitch I, Phillips RL, Springer NM: Profiling expression changes caused by a segmental aneuploid in maize. BMC Genomics 2008, 9:7
11. Stenberg P, Lundberg LE, Johansson AM, Ryden P, Svensson MJ, Larsson J: Buffering of segmental and chromosomal aneuploidies in Drosophila melanogaster. PLoS Genet 2009, 5:e1000465.

12. Ohlsson R: Genetics. Widespread monoallelic expression. Science 2007, 318:1077-1078.

13. Lucchesi JC, Kelly WG, Panning B: Chromatin remodeling in dosage compensation. Annu Rev Genet 2005, 39:615-651.

14. Gupta V, Parisi M, Sturgill D, Nuttall R, Doctolero M, Dudko OK, Malley JD, Eastman PS, Oliver B: Global analysis of X-chromosome dosage compensation. J Biol 2006, 5:3.

15. Nguyen DK, Disteche CM: Dosage compensation of the active $X$ chromosome in mammals. Nat Genet 2006, 38:47-53.

16. Lin H, Gupta V, Vermilyea MD, Falciani F, Lee JT, O'Neill LP, Turner BM: Dosage compensation in the mouse balances up-regulation and silencing of X-linked genes. PLoS Bio/ 2007, 5:e326.

17. Graves JA, Disteche CM: Does gene dosage really matter? J Bio/ 2007, 6:1

18. Arnold AP, Itoh Y, Melamed E: A bird's-eye view of sex chromosome dosage compensation. Annu Rev Genomics Hum Genet 2008, 9:109-127.

19. Mank JE: The $W, X, Y$ and $Z$ of sex-chromosome dosage compensation. Trends Genet 2009, 25:226-233.

20. Charlesworth $B$ : The evolution of chromosomal sex determination and dosage compensation. Curr Biol 1996, 6:149-162.

21. Marin I, Siegal ML, Baker BS: The evolution of dosage-compensation mechanisms. Bioessays 2000, 22:1106-1114.

22. Charlesworth D, Charlesworth B, Marais G: Steps in the evolution of heteromorphic sex chromosomes. Heredity 2005, 95:118-128.

23. Larsson J, Meller VH: Dosage compensation, the origin and the afterlife of sex chromosomes. Chromosome Res 2006, 14:417-431.

24. Marshall Graves JA, Peichel CL: Are homologies in vertebrate sex determination due to shared ancestry or to limited options? Genome Biol 2010, 11:205.

25. Birchler JA, Bhadra U, Bhadra MP, Auger DL: Dosage-dependent gene regulation in multicellular eukaryotes: implications for dosage compensation, aneuploid syndromes, and quantitative traits. Dev Biol 2001, 234:275-288

26. Charlesworth B: Model for evolution of Y chromosomes and dosage compensation. Proc Natl Acad Sci USA 1978, 75:5618-5622.

27. Mukherjee AS, Beermann W: Synthesis of ribonucleic acid by the Xchromosomes of Drosophila melanogaster and the problem of dosage compensation. Nature 1965, 207:785-786.

28. Straub T, Gilfillan GD, Maier VK, Becker PB: The Drosophila MSL complex activates the transcription of target genes. Genes Dev 2005, 19:2284-2288.

29. Hamada FN, Park PJ, Gordadze PR, Kuroda MI: Global regulation of X chromosomal genes by the MSL complex in Drosophila melanogaster. Genes Dev 2005, 19:2289-2294

30. Gelbart ME, Kuroda MI: Drosophila dosage compensation: a complex voyage to the X chromosome. Development 2009, 136:1399-1410.

31. Grimaud C, Becker PB: Form and function of dosage-compensated chromosomes - a chicken-and-egg relationship. BioEssays 2010, 32:709-717.

32. Straub T, Becker PB: Dosage compensation: the beginning and end of generalization. Nat Rev Genet 2007, 8:47-57.

33. Straub T, Becker PB: DNA sequence and the organization of chromosomal domains. Curr Opin Genet Dev 2008, 18:175-180.

34. Robinson PJ, Fairall L, Huynh VA, Rhodes D: EM measurements define the dimensions of the "30-nm" chromatin fiber: evidence for a compact interdigitated structure. Proc Natl Acad Sci USA 2006, 103:6506-6511.

35. Shogren-Knaak M, Ishii H, Sun JM, Pazin MJ, Davie JR, Peterson CL: Histone H4-K16 acetylation controls chromatin structure and protein interactions. Science 2006, 311:844-847.

36. Oliver B: Sex, dose, and equality. PLoS Bio/ 2007, 5:e340.

37. Payer $B$, Lee JT: $X$ chromosome dosage compensation: how mammals keep the balance. Annu Rev Genet 2008, 42:733-772.

38. Zhao J, Sun BK, Erwin JA, Song JJ, Lee JT: Polycomb proteins targeted by a short repeat RNA to the mouse X chromosome. Science 2008, 322:750-756.

39. Maenner S, Blaud M, Fouillen L, Savoye A, Marchand V, Dubois A, Sanglier-Cianférani S, Van Dorsselaer A, Clerc P, Avner P, Visvikis A, Branlant C: 2-D structure of the A region of Xist RNA and its implication for PRC2 association. PLoS Biol, 8:e1000276.

40. Jans J, Gladden JM, Ralston EJ, Pickle CS, Michel AH, Pferdehirt RR, Eisen MB, Meyer BJ: A condensin-like dosage compensation complex acts at a distance to control expression throughout the genome. Genes Dev 2009, 23:602-618. 
41. Cleard F, Spierer P: Position-effect variegation in Drosophila: the modifier Su(var)3-7 is a modular DNA-binding protein. EMBO Rep 2001, 2:1095-1100.

42. Spierer A, Seum C, Delattre M, Spierer P: Loss of the modifiers of variegation Su(var)3-7 or HP1 impacts male X polytene chromosome morphology and dosage compensation. J Cell Sci 2005, 118:5047-5057.

43. Spierer A, Begeot F, Spierer P, Delattre M: SU(VAR)3-7 links heterochromatin and dosage compensation in Drosophila. PLoS Genet 2008, 4:e1000066.

44. Badenhorst P, Voas M, Rebay I, Wu C: Biological functions of the ISWI chromatin remodeling complex NURF. Genes Dev 2002, 16:3186-3198.

45. Deuring R, Fanti L, Armstrong JA, Sarte M, Papoulas O, Prestel M, Daubresse G, Verardo M, Moseley SL, Berloco M, Tsukiyama T, Wu C, Pimpinelli S, Tamkun $J W:$ The ISWI chromatin remodeling protein is required for gene expression and the maintenance of higher order chromatin structure in vivo. Mol Cell 2000, 5:355-365.

46. Corona DF, Siriaco G, Armstrong JA, Snarskaya N, McClymont SA, Scott MP, Tamkun JW: ISWI regulates higher-order chromatin structure and histone $\mathrm{H} 1$ assembly in vivo. PLoS Biol 2007, 5:e232.

47. Buscaino A, Kocher T, Kind JH, Holz H, Taipale M, Wagner K, Wilm M, Akhtar A: MOF-regulated acetylation of MSL-3 in the Drosophila dosage compensation complex. Mol Cell 2003, 11:1265-1277.

48. Morales V, Straub T, Neumann MF, Mengus G, Akhtar A, Becker PB: Functional integration of the histone acetyltransferase MOF into the dosage compensation complex. EMBO J 2004, 23:2258-2268.

49. Robinson PJ, Rhodes D: Structure of the' $30 \mathrm{~nm}^{\prime}$ chromatin fibre: a key role for the linker histone. Curr Opin Struct Bio/ 2006, 16:336-343.

50. Akhtar A, Becker PB: Activation of transcription through histone $\mathrm{H} 4$ acetylation by MOF, an acetyltransferase essential for dosage compensation in Drosophila. Mol Cell 2000, 5:367-375.

51. Prestel M, Feller C, Straub T, Mitlöhner H, Becker PB: The activation potential of MOF is constrained for dosage compensation. Mol Cell 2010, 38:815-826.

52. Mendjan S, Taipale M, Kind J, Holz H, Gebhardt P, Schelder M, Vermeulen M, Buscaino A, Duncan K, Mueller J, Wilm M, Stunnenberg HG, Saumweber H, Akhtar A: Nuclear pore components are involved in the transcriptional regulation of dosage compensation in Drosophila. Mol Cell 2006, 21:811-823.

53. Bienz M: The PHD finger, a nuclear protein-interaction domain. Trends Biochem Sci 2006, 31:35-40.

54. Gilfillan GD, Straub T, de Wit E, Greil F, Lamm R, van Steensel B, Becker PB: Chromosome-wide gene-specific targeting of the Drosophila dosage compensation complex. Genes Dev 2006, 20:858-870.

55. Alekseyenko AA, Peng S, Larschan E, Gorchakov AA, Lee OK, Kharchenko P, McGrath SD, Wang Cl, Mardis ER, Park PJ, Kuroda MI: A sequence motif within chromatin entry sites directs MSL establishment on the Drosophila X chromosome. Cell 2008, 134:599-609.

56. Rea S, Xouri G, Akhtar A: Males absent on the first (MOF): from flies to humans. Oncogene 2007, 26:5385-5394.

57. Li X, Dou Y: New perspectives for the regulation of acetyltransferase MOF. Epigenetics 2010, 5 DOl:10.4161/epi.5.3.11372

58. Gupta A, Sharma GG, Young CS, Agarwal M, Smith ER, Paull TT, Lucchesi JC, Khanna KK, Ludwig T, Pandita TK: Involvement of human MOF in ATM function. Mol Cell Biol 2005, 25:5292-5305.
59. Gupta A, Guerin-Peyrou TG, Sharma GG, Park C, Agarwal M, Ganju RK, Pandita S, Choi K, Sukumar S, Pandita RK, Ludwig T, Pandita TK: The mammalian ortholog of Drosophila MOF that acetylates histone $\mathrm{H} 4$ lysine 16 is essential for embryogenesis and oncogenesis. Mol Cell Biol 2008, 28:397-409.

60. Fraga MF, Ballestar E, Villar-Garea A, Boix-Chornet M, Espada J, Schotta G, Bonaldi T, Haydon C, Ropero S, Petrie K, lyer NG, Pérez-Rosado A, Calvo E, Lopez JA, Cano A, Calasanz MJ, Colomer D, Piris MA, Ahn N, Imhof A, Caldas C, Jenuwein T, Esteller M: Loss of acetylation at Lys 16 and trimethylation at Lys 20 of histone $\mathrm{H} 4$ is a common hallmark of human cancer. Nat Genet 2005, 37:391-400.

61. Pfister S, Rea S, Taipale M, Mendrzyk F, Straub B, Ittrich C, Thuerigen O, Sinn HP, Akhtar A, Lichter P: The histone acetyltransferase hMOF is frequently downregulated in primary breast carcinoma and medulloblastoma and constitutes a biomarker for clinical outcome in medulloblastoma. Int $\mathrm{J}$ Cancer 2008, 122:1207-1213.

62. Kapoor-Vazirani P, Kagey JD, Powell DR, Vertino PM: Role of hMOFdependent histone $\mathrm{H} 4$ lysine 16 acetylation in the maintenance of TMS1/ ASC gene activity. Cancer Res 2008, 68:6810-6821

63. Dou Y, Milne TA, Tackett AJ, Smith ER, Fukuda A, Wysocka J, Allis CD, Chait BT, Hess JL, Roeder RG: Physical association and coordinate function of the H3 K4 methyltransferase MLL1 and the H4 K16 acetyltransferase MOF. Cell 2005, 121:873-885

64. Smith ER, Cayrou C, Huang R, Lane WS, Cote J, Lucchesi JC: A human protein complex homologous to the Drosophila MSL complex is responsible for the majority of histone $\mathrm{H} 4$ acetylation at lysine 16 . Mol Cell Biol 2005, 25:9175-9188.

65. Taipale M, Rea S, Richter K, Vilar A, Lichter P, Imhof A, Akhtar A: hMOF histone acetyltransferase is required for histone $\mathrm{H} 4$ lysine 16 acetylation in mammalian cells. Mol Cell Biol 2005, 25:6798-6810.

66. Li X, Wu L, Corsa CA, Kunkel S, Dou Y: Two mammalian MOF complexes regulate transcription activation by distinct mechanisms. Mol Cell 2009, 36:290-301.

67. Sykes SM, Mellert HS, Holbert MA, Li K, Marmorstein R, Lane WS, McMahon SB: Acetylation of the p53 DNA-binding domain regulates apoptosis induction. Mol Cell 2006, 24:841-851.

68. Fauth T, Muller-Planitz F, Konig C, Straub T, Becker PB: The DNA binding CXC domain of MSL2 is required for faithful targeting the Dosage Compensation Complex to the X chromosome. Nucleic Acids Res 2010, 38:3209-3221.

69. Morra R, Smith ER, Yokoyama R, Lucchesi JC: The MLE subunit of the Drosophila MSL complex uses its ATPase activity for dosage compensation and its helicase activity for targeting. Mol Cell Biol 2008, 28:958-966.

70. Oh H, Parrott AM, Park Y, Lee CG: Regulation of inter- and intramolecular interaction of RNA, DNA, and proteins by MLE. Methods Mol Biol 2010, 587:303-326.

doi:10.1186/gb-2010-11-8-216

Cite this article as: Prestel $M$, et al: Dosage compensation and the global re-balancing of aneuploid genomes. Genome Biology 2010, 11:216. 